\title{
Psychometric Validation of the Persian Self-Compassion Scale Youth Version
}

\author{
Nabi Nazari ${ }^{1}$ (D) $\cdot$ Ronald M. Hernández ${ }^{2} \cdot$ Yolvi Ocaña-Fernandez $^{3} \cdot$ Mark D. Griffiths $^{4}$
}

Accepted: 22 November 2021 / Published online: 7 January 2022

(c) The Author(s), under exclusive licence to Springer Science+Business Media, LLC, part of Springer Nature 2021

\begin{abstract}
Objectives Empirical research investigating self-compassion is a rapidly developing field, and it is potentially crucial in early adolescence. The primary aim of the present study was to psychometrically evaluate the Persian translation of the Self-Compassion Scale Youth version (SCS-Y) and evaluate its factor structure among young adolescents. The second aim was to explore the buffering effect of self-compassion against the negative effect of difficulties in emotion regulation on COVID-19-related anxiety.

Methods A sample of young students ( $n=532$; mean age 13.57 years) completed an online survey, which included the SCS-Y, Patient Health Questionnaire, Difficulties In Emotion Regulation Scale, Coronavirus Anxiety Scale, Youth Life Orientation Test, Brief Resilience Scale, and Brief 10-Item Big Five Inventory. First-order (six-factor) confirmatory factor analysis (CFA) and bi-factor exploratory structural equation modeling (ESEM) analysis were used to evaluate the factor structure of the SCS-Y.

Results Results showed that the SCS-Y had very good internal consistency (Cronbach's alpha coefficient: 0.88; McDonald's omega coefficient: 0.90$)$, composite reliability (0.87), and adequate test-retest reliability after 4 weeks $(0.60)$. The first-order (six-factor) CFA and bi-factor ESEM analysis demonstrated the SCS-Y had excellent dimensionality. Further analysis found negative associations between self-compassion with both depression and neuroticism, and positive associations between self-compassion with both resilience and optimism. Moreover, self-compassion moderated the association between emotion dysregulation and anxiety generated by the COVID-19. Overall, the findings indicated that the SCS-Y had acceptable criterion-related validity, convergent validity, and discriminant validity.

Conclusions The findings provide evidence that the SCS-Y is a reliable and valid instrument for assessing the six factors of self-compassion among younger adolescents. Based on the study's findings, self-compassion appears to be a protective factor against mental health problems during the COVID-19 pandemic for younger adolescents.
\end{abstract}

Keywords Self-compassion · Emotion regulation $\cdot$ Personality $\cdot$ Optimism $\cdot$ Adolescents $\cdot$ COVID-19

For most adolescents, self-criticism, self-blame, and selfnegative evaluation are automatic and habitual responses to rejection or failure associated with psychopathological

Nabi Nazari

Nazariirani@gmail.com

1 Department of Psychology, Faculty of Human Sciences, Lorestan University, Khorramabad, Iran

2 Unidad de Virtualización Académica, Universidad de San Martin de Porres, Lima, Peru

3 Universidad Privada San Juan Bautista, Lima, Peru

4 International Gaming Research Unit, Psychology Division, Nottingham Trent University, Nottingham, UK vulnerability (Cleare et al., 2019). However, self-compassion is related to well-being and is a more adaptive response in times of personal struggle (Di Fabio \& Saklofske, 2020; Neff et al., 2007; Werner et al., 2012). Self-compassion, rooted in Buddhist philosophy, represents the balance between decreased negative and increased positive self-responding (Neff, 2003, 2016). Numerous studies have shown that selfcompassion is associated with positive psychological wellbeing (McKay \& Walker, 2021) and is a protective factor in the development of psychopathology (Wilson et al., 2018) in different cultures (Neff et al., 2019) and genders (Yarnell et al., 2019) among adults.

Regarding adolescents, most research examining selfcompassion has been carried out among older adolescents 
rather than younger adolescents. More specifically, empirical studies indicate that self-compassion among older adolescents is a protective factor against anxiety (Gill et al., 2018), depression (Pullmer et al., 2019), risky behaviors (Marsh et al., 2018), and post-traumatic disorders (Neff \& McGehee, 2010). Also, self-compassion has been associated with resilience (Bluth et al., 2018), emotional well-being (Cunha et al., 2016), and emotion regulation (Moreira \& Canavarro, 2020). Empirical research investigating self-compassion is a rapidly developing field and it is potentially crucial during early adolescence.

Early adolescence is a distinct and complex transitional stage of human growth and development, situated between childhood and later adolescence. Early adolescence is characterized by physical and psychological changes, identity formation, gender conformity, and self-concept development (Roeser \& Pinela, 2014). Also, brain development during early adolescence impacts emotional, cognitive, physical, and mental ability. For example, while emotional reactivity increases, the brain regions related to control are still immature (Morales \& Fox, 2019). Younger adolescents may be more vulnerable to psychopathology and maltreatment. Compared with maltreatment or mental health problems in later adolescence, such experiences occurring in early adolescence can lead to more significant negative effects on mental health and social adjustment (Pechtel \& Pizzagalli, 2011). Therefore, healthy transitions during early adolescence are crucial for health and well-being in adulthood. Compared with older adolescents, younger adolescents are at the incipient stages in the development of the cognitive and emotional resources to regulate emotions. The promotion of adaptive coping strategies, such as self-compassion, during early adolescence may reduce the risk of internalizing disorders in the later stages of adolescence and emerging adulthood (Compas et al., 2017). Growing evidence indicates that self-compassion can develop during childhood and younger adolescents can benefit from self-compassion (Cheang et al., 2019).

Although there is relative lack of evidence among young adolescents, self-compassion has been associated with positive psychological indicators including resilience (Bluth et al., 2016), life satisfaction (Karakasidou et al., 2021), subjective well-being (Bluth \& Blanton, 2015), and executive function proficiency (Shin et al., 2016). Self-compassion is also likely to play an important role in the well-being of younger adolescents given the importance of identity formation and self-concept development during this period (Erikson, 1968). Taken together, the precise assessment of such strategies may be helpful for the diagnosis of emotional problems during early adolescence and for designing more efficacious prevention programs. However, there are far fewer self-compassion studies comprising younger adolescents compared to those with older adolescent and adult samples. The lack of specific instrument that assesses selfcompassion among early adolescents may be one reason for this bias in the published literature.

To date, self-compassion studies have employed the same instruments across different developmental stage groups and have applied adult self-compassion measures to adolescents with little or no modification. For example, the adult Self-Compassion Scale (SCS; Neff, 2003) is a widely used instrument that has also been used to assess self-compassion among adolescents. Younger adolescents may potentially find problems with the concept of SCS. To better understand the concept, the scale and items must be brief, understandable, appropriate to the developmental stage, and be relevant to the experiences of younger adolescents. For an accurate self-compassion assessment, a developmental approach in designing a specific instrument may provide the opportunity to carry out more robust research comprising younger adolescents who are often overlooked in research examining well-being. Moreover, the development of a scale and collection of quantitative data directly from children, rather than relying on the measure for adults, provides more reliable information. The Self-Compassion Scale for Children (SCSC) is a 12-item scale developed to assess self-compassion among children aged 8-12 years old (Sutton et al., 2018). However, this brief scale is arguably narrow in assessing all six dimensions of self-compassion.

The Self-Compassion Scale Youth (SCS-Y) version is a multidimensional scale specifically developed to assess self-compassion and its six dimensions among younger adolescents (Neff et al., 2021). The six dimensions comprise self-kindness, common humanity, self-judgment, isolation, mindfulness, and over-identification. The six dimensions refer to conceptually different strategies and a range of individual responses implicated in struggle situations. All six elements of self-compassion are conceptually separable but comprise an integrated system in which the dimensions interact and influence each other. Self-kindness versus self-judgment refers to a range of individual differences in emotional responses to suffering. Common humanity versus isolation refers to a range of individual cognitive understandings of difficulties and disadvantages. Mindfulness versus over-identification refers to paying attention to painful feelings and thoughts in a mindfully balanced manner rather than over-identified repeated biased attention. Self-compassion theory encourages individuals to embrace their fears, shortcomings, and disappointments as the inherent parts of common humanity so that individuals can be more forgiving and compassionate to themselves and others.

Recently, research into self-compassion has raised issues concerning the factor structure and dimensionality of the adult SCS (Neff, 2003). Self-compassion has typically been assessed as a bi-dimensional construct, comprising the positive dimension or self-warmth (self-kindness, common 
humanity, and mindfulness) and the negative dimension or self-coldness (self-judgment, isolation, over-identification) (Muris et al., 2016; Potter et al., 2014). However, using two scores representing positive (compassionate) responses and negative (uncompassionate) responses is not optimal given that studies have demonstrated poor psychometric properties for the two factors (e.g., Neff et al., 2019). Conceptually, the two-factor approach fails to distinguish between different domains of individual responding (e.g., emotional, cognitive, and attentional). Bi-factor exploratory structural equation modeling (ESEM) has been used to help conceptualize self-compassion as an integrated system with six independent subscales (Neff, 2016). ESEM is a robust psychometric method and has been utilized to overcome the limitations of confirmatory factor analysis (CFA; i.e., all cross-loadings constrained to zero; overestimated correlations between latent constructs) (Marsh et al., 2011). ESEM also incorporates the benefits of exploratory factor analysis (EFA) approach (i.e., cross-loadings) and the CFA approach (i.e., a priori defined structure; Asparouhov \& Muthén, 2009). ESEM is a promising framework representing superior model fit over CFA and a possible alternative to CFA (Asparouhov et al., 2015; Marsh et al., 2014). A bi-factor-ESEM approach provides a more precise psychometric examination in simultaneously evaluating both general factor and sixfactor construct-relevant multidimensionality (Morin et al., 2016). Recent investigations have demonstrated that utilizing the bi-factor-ESEM approach is best when addressing the balance between self-compassion as an integrated system with six independent subscales (Neff et al., 2019; TóthKirály \& Neff, 2020; Tóth-Király et al., 2017). The precise assessment of self-compassion, as a effective coping strategy, may be helpful for the diagnosis of emotional problems during early adolescence and for designing more efficacious prevention programs, particularly during traumatic events.

Exposure to trauma and maltreatment in early adolescence may prevent the development of adaptive emotion regulation skills and self-compassion (Vettese et al., 2011). While difficulty with emotion regulation is associated with internalizing disorders (Li et al., 2021). Lack of adaptive emotion regulation strategies predicts increase in anxiety during the COVID-19 pandemic. In addition, anxiety generated by the COVID-19 can amplify the adverse impacts of the pandemic, such as uncertainty and anxiety sensitivity (Wu et al., 2021). There is growing evidence demonstrating that self-compassion may mitigate the initiation and maintenance of internalizing symptomatology. On the other hand, the relationship between self-compassion and anxiety appears to be well-documented. A higher level of self-compassion can potentially diminish the severity or intensity of negative affective response, emotional reactivity, and adverse psychological consequences in response to daily emotive situations and stressful events, particularly among vulnerable groups such as children (Coyne et al., 2020).

Higher self-compassion can reduce neuroticism by reducing negative self-responding to traumatic life events (Hayes et al., 2016; Li et al., 2021). A longitudinal study of adolescents involved in a traumatic event demonstrated that students with higher self-compassion were protected against later development of both anxiety and depressive symptoms (Zeller et al., 2015). Also, self-compassion has been found to be the main predictor of improvements in perceived stress, rumination, and depressive symptoms among adolescents (Galla, 2016). Regarding exposure to traumatic life events, research has shown that self-compassion protects against anxiety in response to stressors (Neff et al., 2007) and is associated with adaptive responses to negative life events (Braehler \& Neff, 2020).

While adolescents are generally considered to be a population at risk from a mental health point of view, younger adolescents naturally experience more stress, specifically in time of uncertainty such as the outbreak of coronavirus disease 2019 (COVID-19). The physical closing of schools, resulting in considerable social isolation for students, has been considered one of the central factors in developing psychological health issues (e.g., lower social connectedness, higher social isolation, higher psychological distress, and higher depression), all of which can be risk factors for anxiety symptoms (Sekowski et al., 2020). Despite accumulating evidence for the mental health benefits of self-compassion against the COVID-19 pandemic's adverse mental health, there is a dearth of literature examining the role of the selfcompassion during the pandemic across younger adolescents.

The present study was conducted because the psychometric evaluation of a specific instrument to assess selfcompassion among early adolescents is important. The study assessed the reliability and validity of an instrument assessing self-compassion among early adolescents, which captures the core concepts of self-compassion and that is easily read and understood by younger adolescents. The present study was also conducted to contribute to the body of empirical evidence of the benefits of self-compassion during early adolescence. Additional analysis was carried out to examine whether self-compassion moderates the association between emotion dysregulation and COVID-19 anxiety.

\section{Method}

\section{Participants}

A sample of 532 young adolescents (270 boys and 262 girls) recruited in the study. The mean age was 13.57 years $(S D=1.01$, range $12-15$ years). The eligibility criteria comprised being aged between 11 and 15 years, having the 
ability to understand questions, read, and complete a consent form and survey, and having Persian language fluency. Permission by the developer of the SCS-Y to use the scale was granted before data collection. Socioeconomic characteristic (SEC) information was not requested because young adolescents may not have reliable knowledge of their family's SECs.

\section{Procedures}

Transcultural Adaptation of the SCS-Y The English version of the SCS-Y was translated to Persian following international guideline (Beaton et al., 2000). In the first step, the English version of the SCS-Y was translated independently into Persian by two Persian translators. One of the translators was aware of the concepts being examined in the scale (a psychologist). The other translator was neither informed nor aware of the scale concepts and had no psychological background. To synthesize a consensus version, an expert committee reviewed the two versions. Then, an English translator completed a backward translation (Persian-to-English) of the consensual version. There were no major changes made during this cultural adaptation.

Pilot Study In the pre-test stage, 32 students were selected from the target sample to evaluate the consensus translated SYS-Y. The participant debriefing was conducted to identify actual and potential linguistic understanding, grammar, and ambiguity. No significant changes to the scale were needed.

Sampling A convenience sample of the younger adolescent general Persian population was recruited face-to-face and via the internet and utilizing snowball sampling. The teachers collected data during school hours. The teachers were postgraduate and doctoral students in educational psychology, and they were fully aware of how to collect data and obtain digitally informed consent.

Sample Size A priori power analysis for multiple linear regression was calculated using G-Power, using an alpha of 0.05 , a power of 0.80 , Cohen's $f^{2}=0.02$, and self-compassion as a predictor to determine the sample size (Faul et al., 2009). The Cohen's $f^{2}=0.02$ value signifies a small effect size (Cohen, 1988). The desired total sample size was 395 . Finally, 532 participants were recruited in the present study, which allowed for a $15 \%$ loss of data.

\section{Measures}

Self-Compassion Scale Youth Version (SCS-Y; Neff et al., 2021) The 17-item SCS-Y was used to assess self-compassion among adolescents (aged 11 to 15 years). The SCS-Y comprises six subscales (i.e., self-kindness, common humanity, and mindfulness, self-judgment, isolation, and over-identification) with items rated on a five-point scale from 1 (almost never) to 5 (almost always). Scores range from 17 to 85 . A grand mean of the six subscale means was used to calculate a total score. A higher score indicates higher self-compassion. The psychometric properties of the scale are presented in the "Results" section.

Two-Item Patient Health Questionnaire (PHQ-2; Kroenke, Spitzer \& Williams) The PHQ-2 was used to assess depression in the past 2 weeks. Respondents rate the two items (e.g., "Trouble falling or staying asleep, or sleeping too much") on a four-point scale ranging from 0 (not at all) to 3 (nearly every day). Scores range from 0 to 6 . A higher score indicates more severe depression symptoms. The scale demonstrated very good internal consistency in the present study $(\alpha=0.84)$.

Brief 10-Item Big Five Inventory (BFI-10; Rammstedt \& John, 2007) In the present study, two items were used to assess neuroticism (e.g., "I see myself as someone who gets nervous easily"). Respondents rate the items on a scale ranging from 1 (strongly disagree) to 5 (strongly agree). Scores range from 2 to 10 . A higher score indicates a higher level of neuroticism. The scale demonstrated very good internal consistency in the present study $(\alpha=0.86)$.

Test Brief Resilience Scale (BRS; Smith et al., 2008) The sixitem BRS was used to assess adolescent abilities to recover from adversity. Respondents rate the items (e.g., "I usually come through difficult times with little trouble") on a fivepoint scale ranging from 1 (strongly disagree) to 5 (strongly agree). Higher scores indicate greater levels of resilience. The scale demonstrated very good internal consistency in the present study $(\alpha=0.85)$.

Difficulties in Emotion Regulation Scale Short Form (DERS-SF; Kaufman et al., 2016) The 18-item DERS-SF was used to assess emotion dysregulation. Respondents rate the items (e.g., "When I'm upset, I have difficulty focusing on other things") on a five-point scale ranging from 1 (almost never) to 5 (almost always). Scores range from 18 to 90. Higher scores indicate greater difficulty in regulating emotion. The scale demonstrated very good internal consistency in the present study $(\alpha=0.85)$.

Youth Life Orientation Test (YLOT; Ey et al., 2005) The 16-item YLOT was used to assess children's optimism and pessimism. Respondents rate the items (e.g., "I usually expect to have a good day") on a four-point scale ranging from 0 (strongly 
disagree true for me) to 3 (strongly agree true for me). Scores range from 0 to 48 . Higher scores indicate greater positive expectations for future events. The scale demonstrated very good reliability in the present study $(\alpha=0.81)$.

Coronavirus Anxiety Scale (CAS; Lee, 2020) The fiveitem CAS was used to assess COVID-19-related anxiety. Respondents rate the items (e.g., "I had trouble falling or staying asleep because I was thinking about the coronavirus") on a five-point scale ranging from 0 (strongly disagree) to 4 (strongly agree). Scores range from 0 to 20 . A higher score indicates a greater level of COVID-19-related anxiety. The scale demonstrated very good internal consistency in the present study $(\alpha=0.84)$.

\section{Data Analyses}

Descriptive analyses were performed using SPSS version 25 (SPSS Inc., Chicago, IL) with a two-tailed 5\% level of significance. There were no missing values in the assessed variables. Therefore, no imputation method was implemented. Chi-squares and independent $t$-tests investigated the differences between groups (boys and girls). The univariate normality assumption was examined using the values of skewness and kurtosis. Additionally, the multicollinearity issue was checked using the variance inflation factor (VIF) $(1<\mathrm{VIF}<3)$ (Hair et al., 2018).

\section{Construct Validity}

The SCS-Y item responses are ordinal. Therefore, sixfactor first-order CFA model and bi-factor ESEM model were investigated to examine the construct validity of the SCS-Y, using weighted least squares mean-variance adjusted (WLSMV) estimator in Mplus Version 7 (Muthén \& Muthén, 2012). The benchmark suggested by $\mathrm{Hu}$ and Bentler (1999) was considered to evaluate the goodness of fit the models (comparative fit index [CFI] and Tucker-Lewis index [TLI] $>0.95$; standardized root mean square residual [SRMR] and root mean square error of approximation [RMSEA] <0.06). Measurement invariance was evaluated across gender (girls/boys) for the superior model. The models were compared utilizing better goodness of fits, higher factor loadings, and lower values of the Bayesian information criterion. The superior CFA model was evaluated across both girls' and boys' samples. Once the quality of the respective models was established, multigroup analysis was conducted with four models, including more constrained models that evaluated configural invariance, metric invariance, scalar invariance, and error variance invariance. A change in CFA $(\triangle C F I)<0.01$, a change in TLI $(\triangle T L I)<0.01$, and a change in RMSEA $(\triangle \mathrm{RMSEA})<0.015$ indicate non-invariance between models (Chen, 2007; Cheung \& Rensvold, 2002). Moreover, the guideline was used to compare whether there were significant differences in the two models' quality.

\section{Reliability}

Reliability was assessed with several of indices. The values of Cronbach's coefficient $>0.8$, McDonald's modelbased composite reliability $(\mathrm{CR})>0.7$, and McDonald's $\omega$ of the total SCS-Y $>0.7$ were deemed to be very good psychometric properties. The test-retest analysis was conducted 4 weeks from the validation study, and reliability was calculated utilizing the intraclass coefficient (ICC). Convergent validity was tested for SCS-Y and its subscales utilizing average extracted variance (AVE $>0.5)$ (Hair et al., 2018). Discriminant validity was checked using Fornell-Larcker criterion. It compares the square root of the AVE with the correlations of latent construct. Statistically, the square root of each construct's AVE values should be greater than its highest correlations with other latent structures (Henseler et al., 2016).

SEM analysis was performed using AMOS version 24 to establish criterion-related validity of SCS-Y. To evaluate criterion-related validity of the SCS-Y and its six subscales, correlation analyses were performed using the scores on the scales assessing depression, neuroticism, resilience, and optimism. It was expected that the SCS-Y, self-kindness, mindfulness, and common humanity would be (i) positively associated with resilience and optimism, and (ii) negatively associated with depression and neuroticism. As for discriminant validity, it was also expected that self-judgment, over-identification, and isolation would be (i) positively associated with depression and neuroticism, and (ii) negatively associated with resilience and optimism. $Z$ test was performed to compare the correlation coefficient of resilience and optimism with self-compassion.

\section{Moderation Analyses}

SEM was conducted to confirm if overall individual abilities in the six dimensions of self-compassion would moderate the effects of emotion dysregulation on COVID-19 anxiety. The interaction effects of maladaptive emotion and self-compassion (maladaptive emotion $\times$ self-compassion) on COVID-19 anxiety were also investigated. An indirect effect is statistically 
significant when the calculated $95 \%$ bias-corrected confidence interval (CI) does not include zero (Hayes, 2017). The 95\%

Table 1 Demographic characteristics of the sample $(N=532)$

\begin{tabular}{llll}
\hline Item & Value & Test & $p$-value \\
\hline Categorical variables & & & \\
Gender, $\boldsymbol{n}$ (\%) & & & .73 \\
Girl & $270(50.8)$ & $\chi^{2}=.12$ & \\
Boy & $262(49.2)$ & & \\
Grade, $\boldsymbol{n}$ (\%) & & & \\
7th & $181(34)$ & &. .24 \\
8th & $191(35.9)$ & $\chi^{2}=2.83$ & \\
9th & $160(30.1)$ & & .54 \\
Continues variables $\boldsymbol{M}(\mathbf{S D})$ & & \\
Age & $13.57(1.01)$ & $t(1,530)=.60$ & \\
Self-compassion youth & $2.51(0.65)$ & $t(1,530)=2.68$ & $<.01$ \\
COVID-19 anxiety & $10.35(3.70)$ & $t(1,530)=-2.04$ & .03 \\
Emotion dysregulation & $41.16(13.92)$ & $t(1,530)=1.75$ & .059 \\
Depression & $1.85(.95)$ & $t(1,530)=-1.73$ & .052 \\
Neuroticism & $5.36(1.92)$ & $t(1,530)=-2.56$ & $<.01$ \\
Resilience & $2.43(0.53)$ & $t(1,530)=2.53$ & $<.01$ \\
Optimism & $12.29(3.11)$ & $t(1,530)=1.65$ & .60 \\
\hline
\end{tabular}

Note: $n$ frequency; $y$ years; $M$ mean; $S D$ standard deviation

$t=$ independent $t$-test to compare gender; negative $t$ value $=$ girls obtained higher score
CI was generated by the bias-corrected method for the point estimate with 5000 bootstrapped samples.

\section{Results}

\section{Descriptive Characteristics at Baseline}

The descriptive characteristics of the sample are presented in Table 1. For all 17 items of SCS-Y, the absolute values of skewness and kurtosis and the VIF values were in the acceptable range $(1<V I F<3)$. Overall, there was no violation according to normality and multicollinearity assumptions (see Table 2). Boys obtained higher scores in self-compassion and resilience than girls. Girls obtained higher scores in neuroticism and COVID-19 anxiety. Significant gender differences were found for self-compassion $(t[530]=2.69$, $p<0.01$, Cohen's $d=0.31,95 \%$ CI [0.17, 0.49], Cohen's $d=0.38,95 \%[0.21,0.53])$ and resilience $(t[530]=2.53$, $p<0.01$, Cohen's $d=0.29,95 \%$ CI [0.15, 0.46] for boys, and neuroticism $(t[530]=2.65, p<0.01$, Cohen's $d=0.35$, $95 \%$ CI $[0.19,0.51])$ and COVID-19 anxiety $(t[530]=2.04$, $p=0.03$, Cohen's $d=0.15,95 \%$ CI $[0.04,0.25]$ ), for girls. No other gender differences were found on any other variables (see Table 1).

Table 2 Item analysis of the SCS-Y $(N=532)$

\begin{tabular}{|c|c|c|c|c|c|c|c|c|c|c|c|c|c|}
\hline & \multirow{2}{*}{$\begin{array}{l}\text { CFA } \\
\text { SF }\end{array}$} & \multicolumn{7}{|c|}{ Bi-factor ESEM } & \multicolumn{5}{|l|}{ Item analysis } \\
\hline & & $\mathrm{SC}$ & $\mathrm{CH}$ & SJ & ISO & SK & MI & OI & Correlation & Cronbach's & Skewness & Kurtosis $^{\mathrm{a}}$ & VIF \\
\hline SK1 & .796 & .828 & .167 & .142 & .238 & .854 & .052 & .096 & .558 & .868 & .770 & -.701 & 1.49 \\
\hline SK2 & .787 & .875 & .146 & .169 & .218 & .812 & .157 & .015 & .534 & .867 & -.034 & -.667 & 1.54 \\
\hline SK3 & .806 & .894 & .168 & .214 & .218 & .791 & .033 & .054 & .519 & .866 & .369 & -.686 & 2.33 \\
\hline SJ1 & .825 & .922 & .219 & .845 & .155 & .202 & .019 & .067 & .634 & .861 & .027 & -1.361 & 1.54 \\
\hline SJ2 & .767 & .765 & .197 & .802 & .148 & .153 & .058 & .116 & .537 & .863 & .057 & -1.31 & 1.58 \\
\hline SJ3 & .867 & .943 & .168 & .870 & .133 & .145 & .089 & .013 & .593 & .862 & -.249 & -.471 & 1.58 \\
\hline $\mathrm{CH} 1$ & .801 & .828 & .871 & .188 & .166 & .132 & .081 & .019 & .586 & .864 & .082 & -.580 & 2.66 \\
\hline $\mathrm{CH} 2$ & .810 & .875 & .888 & .151 & .122 & .175 & .007 & .052 & .572 & .866 & .046 & -.814 & 2.66 \\
\hline $\mathrm{CH} 3$ & .868 & .829 & .867 & .249 & .143 & .152 & .031 & .065 & .626 & .863 & .785 & -.235 & 2.76 \\
\hline IS1 & .858 & .922 & .165 & .120 & .849 & .262 & .043 & .062 & .335 & .876 & .598 & -.508 & 2.19 \\
\hline IS2 & .756 & .765 & .154 & .143 & .845 & .233 & .065 & .029 & .463 & .874 & 1.19 & 1.11 & 1.79 \\
\hline IS3 & .624 & .828 & .111 & .169 & .797 & .153 & .107 & .075 & .512 & .873 & .434 & -.645 & 2.18 \\
\hline MI1 & .865 & .828 & -.007 & .053 & .044 & .105 & .815 & -.015 & .683 & .864 & -.083 & -.980 & 1.33 \\
\hline MI2 & .874 & .875 & .046 & .032 & .066 & .114 & .856 & .048 & .676 & .863 & .577 & -.448 & 2.35 \\
\hline MI3 & .901 & .829 & .059 & .052 & .068 & -.028 & .819 & .017 & .707 & .863 & .275 & -.596 & 1.58 \\
\hline OI1 & .846 & .875 & .043 & .066 & .060 & .070 & .014 & .933 & .606 & .870 & .416 & -.536 & 2.25 \\
\hline OI2 & .798 & .829 & .082 & .113 & .096 & .075 & .041 & .917 & .638 & .868 & -.047 & -.825 & 2.49 \\
\hline
\end{tabular}

Note: $C F A$ confirmatory factor analysis; Bi-factor ESEM bi-factor exploratory structural equation modeling; $S K$ self-kindness; $S J$ self-judgment (reverse-coded); $\mathrm{CH}$ common humanity; $I S$ isolation (reverse-coded); $M I$ mindfulness; $O I$ over-identification (reverse-coded); $S C$ self-compassion general factor; significant target loadings in bold 


\section{Construct Validity}

Evaluation of the fit indices showed that the six-factor firstorder CFA model $\left(\chi^{2} / \mathrm{df}=2.68, \mathrm{CFI}=0.965, \mathrm{SRMR}=0.048\right.$, RMSEA $=0.056,90 \%$ CI [0.049, 0.064]), and bi-factor ESEM model $\left(\chi^{2} / \mathrm{df}=1.45, \mathrm{CFI}=0.990, \mathrm{SRMR}=0.029\right.$, RMSEA $=0.03,90 \%$ CI $[0.016,0.046]$ ) fitted the data well. Also, comparison of both models found superior fit for the bi-factor solution. The standardized factor loadings for the models showed that all 17 SCS-Y items loaded significantly on their specific factors, factor loading $>0.30$ (see Table 2). The bi-factor ESEM model demonstrated excellent goodness of fit. Moreover, the bi-factor ESEM loading factors were higher than loading factors for six-factor CFA model. Measurement invariance was conducted across gender for the bifactor ESEM. The analysis produced excellent fit for both the boy sample $\left(\chi^{2} / \mathrm{df}=1.19, \mathrm{CFI}=0.996, \mathrm{SRMR}=0.027\right.$, RMSEA $=0.01,90 \%$ CI [0.001, 0.034]), and girl sample $\left(\chi^{2} /\right.$ $\mathrm{df}=1.24, \mathrm{CFI}=0.991, \mathrm{SRMR}=0.033$, RMSEA $=0.031$, $90 \%$ CI [0.001, 0.048]). Finally, the results of the multigroup CFA analysis are presented in Table $3(\triangle T L I<0.01$, $\triangle C F I<0.01)$. The measurement invariance analysis indicated that the SCS-Y was fully invariant across gender.

\section{Reliability}

The values of Cronbach's alpha ( $\alpha=0.88)$, McDonald's $(\omega=0.90)$, and McDonald's model-based composite reliability $(\mathrm{CR}=0.87)$ of the total SCS-Y were all satisfactory (see Table 4). The Cronbach's alpha if item deleted values (higher than 0.7) and the squared multiple correlation values (higher than 0.3) were all well above the recommended levels (see Table 2). After 4 weeks of the validation study, the SCS-Y was sent to sample who were randomly selected from the study sample by a random number generator $(n=250)$. Of these, 122 surveys were received. The ICC was 0.60 with $95 \%$ CI $[0.40,0.74], F(1,121)=2.16, p<0.001)$. The AVE value was higher than 0.50 for SCS-Y (0.67) and for each SCS-Y dimension (ranging between 0.566 and 0.775). Moreover, the following relationship was obtained: $\mathrm{MSV}<\mathrm{AVE}<\mathrm{CR}<\operatorname{MaxR}(\mathrm{H})$. Furthermore, the squared root of AVE values was higher than the coefficient of the correlation between factors (see Table 4).

The SEM analysis found negative associations between self-compassion with both depression and neuroticism and positive associations between self-compassion with both resilience and optimism (demonstrating convergent validity). Also, self-compassion was more significantly associated with resilience $(Z$ score $=2.49 ; p=0.007)$, compared with optimism (demonstrating discriminant validity). It
Table 3 Invariance measurement $(N=532)$

\begin{tabular}{ccccccccc}
\hline Model & Invariance type & $\chi^{2} / d f$ & CFI & $\Delta$ CFI & TLI & $\Delta$ TLI & AIC & RMSEA 90\% [CI] \\
\hline Bi-factor ESEM & & & & & & & \\
Configural & 1.331 & .992 & -- & .986 & -- & 549.7 & $.020[.005, .029]$ \\
Weak (metric) & 1.413 & .984 & -.008 & .978 & -.008 & 554.3 & $.026[.018, .034]$ \\
& Strong (scalar) & 1.453 & .982 & -.010 & .976 & -.010 & 549.3 & $.027[.017, .034]$ \\
Strict & 1.390 & .983 & -.009 & .980 & -.006 & 522.4 & $.025[.017, .032]$ \\
\hline
\end{tabular}

Note: $C F I$ comparative fit index; TLI Tucker-Lewis index; RMSEA root mean square error of approximation (RMSEA); $C I$ confidence interval; AIC Akaike information criterion

Table 4 Validity analysis and correlations between SCS-Y factors

\begin{tabular}{|c|c|c|c|c|c|c|c|c|c|c|c|c|c|}
\hline Item & Mean & SD & $\alpha$ & MSV & AVE & $\mathrm{CR}$ & $\operatorname{MaxR}(\mathrm{H})$ & 1 & 2 & 3 & 4 & 5 & 6 \\
\hline 1. Self-kindness & 2.63 & .83 & .82 & .413 & .634 & .839 & .84 & $.796 * * *$ & & & & & \\
\hline 2. Self-judgment & 2.51 & .98 & .85 & .417 & .674 & .861 & .87 & $.641 * *$ & $.821 * * *$ & & & & \\
\hline 3. Common humanity & 3.02 & .70 & .86 & .417 & .683 & .88 & .871 & $.506 * *$ & $.646 * * *$ & $.827 * * *$ & & & \\
\hline 4. Isolation & 2.60 & .98 & .82 & .061 & .566 & .83 & .841 & $.162 * *$ & $.221 * * *$ & $.246 * * *$ & $.752 * * *$ & & \\
\hline 5. Mindfulness & 3.44 & 1.06 & .90 & .290 & .775 & .91 & .913 & $.353^{* *}$ & $.538 * * *$ & $.393 * * *$ & $.224 * * *$ & $.88 * * *$ & \\
\hline 6. Over-identification & 2.43 & .81 & .807 & .413 & .676 & .81 & .81 & $.643 * *$ & $.504 * * *$ & $.438 * * *$ & $.239 * * *$ & $.296 * * *$ & $.822 * * *$ \\
\hline
\end{tabular}

Note: In bold: squared root of the AVE

$\mathrm{MSV}<\mathrm{AVE}<\mathrm{CR}<\operatorname{MaxR}(\mathrm{H})$, and $.5<\mathrm{CR}$

$C R$ composite reliability; $S D$ standard deviation; $A V E$ average extracted variance; $M S V$ maximum shared variance; $\operatorname{Max} R(H)$ maximum reliability

${ }^{* * *} p<.001$ 


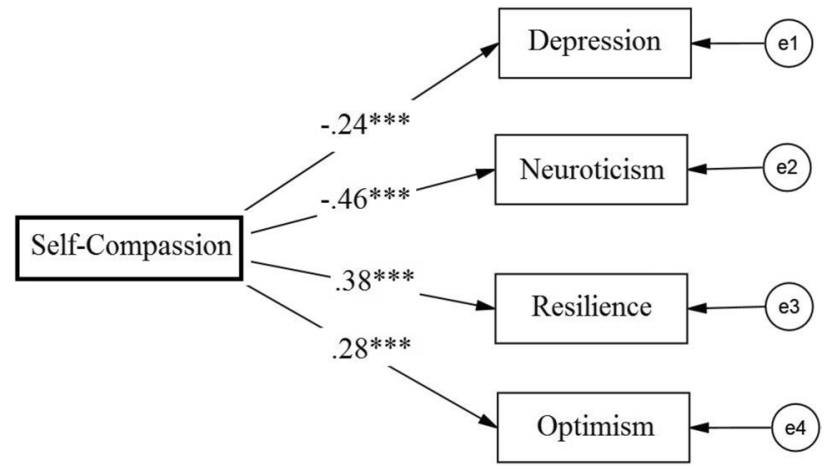

Fig. 1 Associations between self-compassion and well-being indicators

Table 5 The SCS-Y dimension bivariate correlations with interested variables

\begin{tabular}{lllll}
\hline Item & Depression & Neuroticism & Resilience & Optimism \\
\hline Self-kindness & $-.37^{* *}$ & $-.32^{* *}$ & $.42^{* *}$ & $.30^{* *}$ \\
Self-judgment & $.32^{* *}$ & $.44^{* *}$ & $-.39^{* *}$ & $-.24^{* *}$ \\
Common human- & $-.22^{* *}$ & $-.14^{* *}$ & $.24^{* *}$ & $.22^{* *}$ \\
$\quad$ ity & & & & \\
Isolation & $.28^{* *}$ & $.25^{* *}$ & $-.32^{* *}$ & $-.29^{* *}$ \\
Mindfulness & $-.34^{* *}$ & $-.39^{* *}$ & $.38^{* *}$ & $.27^{* *}$ \\
$\begin{array}{l}\text { Over-identifica- } \\
\quad \text { tion }\end{array}$ & $.22^{* *}$ & $.16^{* *}$ & $-.23^{* *}$ & $-.18^{* *}$ \\
\hline
\end{tabular}

Note: $* p<.05 ; * * p<.01$

was also found that self-compassion predicted neuroticism $(\beta=-0.46, S E=0.03, p<0.001)$, depression $(\beta=-0.24$, $S E=0.04, p<0.001)$, resilience $(\beta=0.38, S E=0.04$, $p<0.001)$, and optimism $(\beta=0.28, S E=0.04, p<0.0 .01)$ (see Fig. 1). Moreover, the six dimensions of self-compassion were significantly correlated with depression, neuroticism, resilience, and optimism. More specifically, each positive mechanism of self-compassion was positively associated with resilience and optimism and each negative mechanism of self-compassion was negatively associated with depression, COVID-19 anxiety, and neuroticism (see Table 5).

\section{Moderation Analyses}

The moderation model is shown in Fig. 2. The results suggested the model fitted the data well; $\chi^{2} / \mathrm{df}=1.00$, $\mathrm{CFI}=1.00, \mathrm{TLI}=1.00, \mathrm{RMSEA}=0,90 \% \mathrm{CI}[0.0,0.04]$. The standardized total effect of emotion dysregulation on COVID-19 anxiety was statistically significant, with a large effect size; $\beta=0.54, S E=0.05, p<0.001, t=7.91$, Cohens $f^{2}=0.42,95 \%$ CI $[0.46,0.61]$. The moderation analysis showed emotion dysregulation directly predicted COVID-19 anxiety with low to moderate effect size: Cohen's $f^{2}=0.09,95 \%$ CI $[0.15,0.39]$. Moreover, self-compassion directly predicted COVID-19 anxiety but with a low effect size; Cohen's $f^{2}=0.04,95 \%$ CI $\left.[0.02,0.09]\right)$. The interaction effect of emotion dysregulation and self-compassion (emotion dysregulation $\times$ self-compassion) significantly predicted COVID-19 anxiety with medium effect size: Cohen's $f^{2}=0.12,95 \%$ CI $[-0.37,-0.21]$.

\section{Discussion}

The primary aim of the present study was to translate the Self-Compassion Scale Youth version to Persian and assess its validity and reliability among younger adolescents. The factor structure analysis suggested that the SCS-Y is a sixfactor construct, and therefore the findings were consistent with the original psychometric validation study (i.e., Neff et al., 2021). The present study provides further empirical evidence of the psychometric robustness of the SCS-Y and its factor structure, using the novel bi-factor ESEM framework, and the testing of measurement invariance. The study's findings provide evidence of the superiority of the bi-factor ESEM of self-compassion compared to first-order CFA (six-factor). Bi-factor ESEM reflected the excellent dimensionality of self-compassion. Findings correspond to

Fig. 2 Moderation analysis

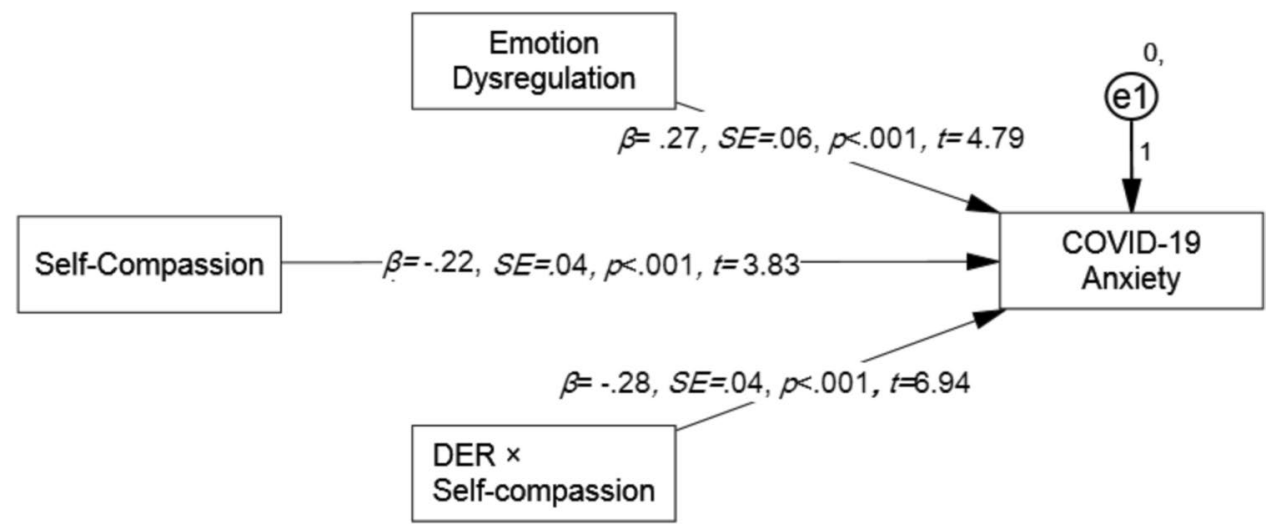


the growing evidence that self-compassion and its six components can be fully understood within a multidimensionality framework that captures construct-relevant psychometric (e.g., Tóth-Király \& Neff, 2020; Tóth-Király et al., 2017). Also, the measurement invariance for bi-factor-ESEM structure yielded further support for the structure stability across gender, which was not evaluated in the original study. The measurement invariance analysis indicated that the SCS-Y was fully invariant across gender. Therefore, the SCS-Y can be used to make reliable comparisons between gender (girls/ boys); the items in the SCS-Y were interpreted the same whether the respondent is boy or girls.

The values of Cronbach's alpha, CR, and McDonald's $\omega$ demonstrated that the Persian SCS-Y had very good internal consistency. In line with research including older adolescents and adults, self-compassion was associated with positive psychological constructs which have previously been identified in the extant literature. This includes resilience (Neff \& McGehee, 2010; Trompetter et al., 2017) and optimism (Muris et al., 2020; Shapira \& Mongrain, 2010). Self-compassion was negatively associated with other constructs identified in the literature including depression (Barlow et al., 2017; Lathren et al., 2019; Raes, 2011) and neuroticism (Pyszkowska, 2020). Also, the positive SCS-Y subscales (self-kindness, common humanity, and mindfulness) were significantly and positively associated with resilience and optimism (Mona \& Angela, 2018), and significantly and negatively associated with depression, emotion dysregulation, COVID-19 anxiety, and neuroticism. Also, the higher scores on the self-judgment, over-identification, and isolation subscales of the SCS-Y were associated positively with higher scores for depression, emotion dysregulation, COVID19 anxiety, and neuroticism (Dreisoerner et al., 2020), and they were associated with lower scores on resilience and optimism. Moreover, the findings support the convergent and discriminant validity for self-compassion construct. Overall, the study's findings indicate that the SCS-Y is a reliable and valid instrument to assess self-compassion across six subscales among the general younger adolescent population.

Significant gender differences were also found. Consistent with research among adults (e.g., Yarnell et al., 2015), boys in the present study reported higher total self-compassion scores than girls. The study findings align with previous studies at a global level indicating that females are more likely to develop anxiety symptoms and be more neurotic than males (Schmitt et al., 2016). In addition, Iranian females (from an eastern culture) reported more psychological problems (e.g., anxiety) associated with COVID-19 than males, which also concurs with previous research (e.g., Nazari et al., 2021; Ahorso et al., 2020). Previous findings also suggest that culture shapes the way emotions are experienced and expressed (von Suchodoletz \& Hepach, 2021). Therefore, one possible explanation of gender differences may relate to cultural contexts (Khramtsova \& Chuykova, 2016; Neff et al., 2008; Pfabigan et al., 2018). For example, in Eastern cultures, individuals are encouraged to dampen their negative emotions, particularly females (Lim, 2016).

The second aim of the study was to explore the buffering effect of self-compassion (as assessed using the SCS-Y) against the negative effect of maladaptive emotion regulation strategies on COVID-19 anxiety. The moderation analysis indicated the buffering effect of self-compassion in the association between emotion dysregulation and anxiety generated by COVID-19. The impact of high state emotion dysregulation on anxiety generated by COVID-19 was lower for adolescents with high self-compassion than for individuals with lower levels of self-compassion. There is evidence that self-compassion potentially buffers the adverse mental health impacts of COVID-19 (Lau et al., 2020). The study offers insights into the way that self-compassion may work to weaken the associations between emotion dysregulation with anxiety generated by COVID-19. The findings suggest the potential positive influence of self-compassion in therapeutic programs designed to reduce the adverse effect of the COVID-19 pandemic, particularly among female adolescents with higher emotion dysregulation. The mechanisms involved with emotion regulation appear to be related to selfcompassion, mainly being aware and accepting emotions, managing emotional impulses, and having strategies to deal with distressing emotions (Inwood \& Ferrari, 2018; Shattell \& Johnson, 2018).

Self-compassion negatively predicted anxiety generated by COVID-19 among younger adolescents in the present study. The findings are in line with an increasing number of studies showing a negative association between selfcompassion and psychopathology vulnerability in the form of anxiety (Pérez-Aranda et al., 2021; Werner et al., 2012). While self-compassion predicts optimism and resilience, there is evidence indicating that traits related to optimism, such as self-compassion, facilitate the recovery from the impacts of trauma and other mental health symptoms (Nazari \& Griffiths, 2020). Self-compassion and its six subscales may address the different ways that individuals emotionally respond to suffering (with kindness or judgment), cognitively understand their predicament (as part of the human experience or as isolating), and the way individuals pay attention to pain (in a mindful or over-identified manner) (Allen \& Leary, 2010; Zeller et al., 2015).

\section{Limitations and Future Research Directions}

The findings of the study should be interpreted in light of several limitations. The study was conducted during the COVID-19 pandemic, and to minimize infection risk, data collection occurred online. Using an online data collection method may limit specific relevant population groups (e.g., 
disadvantaged groups) and other vulnerable groups. Therefore, the data cannot represent the views of these disadvantaged groups, and affects the study findings' generalizability. However, online data collection tends to provide more honest and truthful responses than those utilizing offline methods (Griffiths, 2010). Another limitation of the present study was that the data relied entirely on self-report measures which have well established methodological biases. Finally, while this study provided novel insight into the relationship between self-compassion and other variables among younger adolescents, cross-sectional studies do not provide evidence of a temporal relationship in relation to these variables. Longitudinal data would be required to confirm the causal nature of the concepts investigated and cross-lagged panel analysis is warranted. For future research, validation of the specific multidimensional research instrument based on the development stage is a promising approach in assessing self-compassion and supporting professionals in reducing the burden of mental health problems among adolescents.

\section{Acknowledgements None.}

Author Contribution NN: conceptualization; methodology; writingoriginal draft preparation. MDG: data curation, writing — revision draft preparation, validation, supervision. RMH \& YOF: visualization, investigation, software. All authors: writing-reviewing and editing.

Data Availability The data that support the findings of this study are available on request from the corresponding author.

\section{Declarations}

Ethics Approval and Consent to Participate The study was performed in accordance with the Declaration of Helsinki and was approved and registered by the ethical and research committees from the following collaborating center. The study was reviewed and approved by the Ethics Committee of Hakim University (IR.IAU.S.REC.1399.006) prospectively. All participants provided a signed written consent.

Competing Interests The authors declare no competing interests.

\section{References}

Ahorsu, D. K., Lin, C. Y., Imani, V., Saffari, M., Griffiths, M. D., \& Pakpour, A. H. (2020). The Fear of COVID-19 Scale: Development and initial validation. International journal of mental health and addiction, 1-9. Advance online publication. https://doi.org/ 10.1007/s11469-020-00270-8

Allen, A. B., \& Leary, M. R. (2010). Self-compassion, stress, and coping. Social and Personality Psychology Compass, 4(2), 107-118. https://doi.org/10.1111/j.1751-9004.2009.00246.x

Asparouhov, T., \& Muthén, B. (2009). Exploratory structural equation modeling. Structural Equation Modeling, 16(3), 397-438. https:// doi.org/10.1080/10705510903008204

Asparouhov, T., Muthén, B., Morin, A. J., Bayesian, S., structural equation modeling with cross-loadings and residual covariance:
Comments on Stromeyer, et al. (2015). Journal of Management, 41(6), 1561-1577. https://doi.org/10.1177/0149206315591075

Barlow, M. R., Goldsmith Turow, R. E., \& Gerhart, J. (2017). Trauma appraisals, emotion regulation difficulties, and self-compassion predict posttraumatic stress symptoms following childhood abuse. Child Abuse and Neglect, 65, 37-47. https://doi.org/10.1016/j. chiabu.2017.01.006

Beaton, D. E., Bombardier, C., Guillemin, F., \& Ferraz, M. B. (2000). Guidelines for the process of cross-cultural adaptation of selfreport measures. Spine, 25(24), 3186-3191. https://doi.org/10. 1097/00007632-200012150-00014

Bluth, K., \& Blanton, P. W. (2015). The influence of self-compassion on emotional well-being among early and older adolescent males and females. Journal of Positive Psychology, 10, 219-230. https:// doi.org/10.1080/17439760.2014.936967

Bluth, K., Campo, R. A., Futch, W. S., \& Gaylord, S. A. (2016). Age and gender differences in the associations of self-compassion and emotional well-being in a large adolescent sample. Journal of Youth and Adolescence, 46(4), 840-853. https://doi.org/10.1007/ s10964-016-0567-2

Bluth, K., Mullarkey, M., \& Lathren, C. (2018). Self-compassion: A potential path to adolescent resilience and positive exploration. Journal of Child and Family Studies, 27, 3037-3047. https://doi. org/10.1007/s10826-018-1125-1

Braehler, C. \& Neff, K. (2020). Self-compassion in PTSD. In M. Tull and N. Kimbrel (Eds.), Emotion in posttraumatic stress disorder (pp. 567-596). Amesterdam: Elsevier Academic Press.

Chen, F. F. (2007). Sensitivity of goodness of fit indexes to lack of measurement invariance. Structural Equation Modeling, 14(3), 464-504. https://doi.org/10.1080/10705510701301834

Cheung, G. W., \& Rensvold, R. B. (2002). Evaluating goodness-of-fit indexes for testing measurement invariance. Structural Equation Modeling, 9(2), 233-255. https://doi.org/10.1207/S19338007S EM0902_5

Cheang, R., Gillions, A., \& Sparkes, E. (2019). Do mindfulnessbased interventions increase empathy and compassion in children and adolescents: A systematic review. Journal of Child and Family Studies, 28(7), 1765-1779. https://doi.org/10.1007/ s10826-019-01413-9

Cleare, S., Gumley, A., \& O'Connor, R. C. (2019). Self-compassion, self-forgiveness, suicidal ideation, and self-harm: A systematic review. Clinical Psychology \& Psychotherapy, 26(5), 511-530. https://doi.org/10.1002/cpp.2372

Cohen, J. (1988). Statistical power analysis for the behavioral sciences (second ed.). London: Routledge. https://doi.org/10.4324/97802 03771587

Compas, B. E., Jaser, S. S., Bettis, A. H., Watson, K. H., Gruhn, M. A., Dunbar, J. P., et al. (2017). Coping, emotion regulation, and psychopathology in childhood and adolescence: A meta-analysis and narrative review. Psychological Bulletin, 143(9), 939-991. https://doi.org/10.1037/bul0000110

Coyne, L. W., Gould, E. R., Grimaldi, M., Wilson, K. G., Baffuto, G., \& Biglan, A. (2020). First things first: Parent psychological flexibility and self-compassion during COVID-19. Behavior Analysis in Practice. Advance online publication. https://doi.org/10.1007/ s40617-020-00435-w

Cunha, M., Xavier, A., \& Castilho, P. (2016). Understanding self-compassion in adolescents: Validation study of the Self-Compassion Scale. Personality and Individual Differences, 93, 56-62. https:// doi.org/10.1016/j.paid.2015.09.023

Di Fabio, A., \& Saklofske, D. H. (2020). The relationship of compassion and self-compassion with personality and emotional intelligence. Personality and Individual Differences, 169(1), 110109. https://doi.org/10.1016/j.paid.2020.110109

Dreisoerner, A., Junker, N. M., \& van Dick, R. (2020). The relationship among the components of self-compassion: A pilot study using 
a compassionate writing intervention to enhance self-kindness, common humanity, and mindfulness. Journal of Happiness Studies, 22, 21-47. https://doi.org/10.1007/s10902-019-00217-4

Erikson, E. H. (1968). Identity: Youth and crisis. Norton.

Ey, S., Hadley, W., Allen, D. N., Palmer, S., Klosky, J., Deptula, D., Thomas, J., \& Cohen, R. (2005). A new measure of children's optimism and pessimism: The youth life orientation test. Journal of Child Psychology and Psychiatry, 46(5), 548-558. https://doi. org/10.1111/j.1469-7610.2004.00372.x

Faul, F., Erdfelder, E., Lang, A.-G., \& Buchner, A. (2009). Statistical power analyses using G*Power 3.1: Tests for correlation and regression analyses. Behavior Research Methods, 41(4), 1149.

Galla, B. M. (2016). Within-person changes in mindfulness and selfcompassion predict enhanced emotional wellbeing in healthy, but stressed adolescents. Journal of Adolescence, 49, 204-217. https://doi.org/10.1016/j.adolescence.2016.03.016

Gill, C., Watson, L., Williams, C., \& Chan, S. W. Y. (2018). Social anxiety and self-compassion in adolescents. Journal of Adolescence, 69(2019), 163-174. https://doi.org/10.1016/j.adole scence.2018.10.004

Griffiths, M. D. (2010). The use of online methodologies in data collection for gambling and gaming addictions. International Journal of Mental Health and Addiction, 8(1), 8-20. https:// doi.org/10.1007/s11469-009-9209-1

Hair, J. F., Black, W. C., Babin, B. J. \& Anderson, R. E. (2018). Multivariate data analysis (8th ed.), Andover, UK: Cengage Learning, https://doi.org/10.1002/9781119409137.ch4.

Hayes, A. F. (2017). Partial, conditional, and moderated moderated mediation: Quantification, inference, and interpretation. Communucation Monographs, 85(1), 4-40. https://doi.org/10.1080/ 03637751.2017 .1352100

Hayes, J. A., Lockard, A. J., Janis, R. A., \& Locke, B. D. (2016). Construct validity of the self-compassion scale-short form among psychotherapy clients. Counselling Psychology Quarterly, 29(4), 405-422. https://doi.org/10.1080/09515070.2016.1138397

Henseler, J., Hubona, G., \& Ray, P. A. (2016). Using PLS path modeling in new technology research : Updated guidelines. Industrial Management \& Data Systems, 116(1), 2-20. https://doi.org/10. 1108/IMDS-09-2015-0382

Hu, L. T., \& Bentler, P. M. (1999). Cutoff criteria for fit indexes in covariance structure analysis: Conventional criteria versus new alternatives. Structural Equation Modeling, 6(1), 1-55. https:// doi.org/10.1080/10705519909540118

Inwood, E., \& Ferrari, M. (2018). Mechanisms of change in the relationship between self-compassion, emotion regulation, and mental health: A systematic review. Applied Psychology: Health and Well-Being, 10(2), 215-235. https://doi.org/10.1111/aphw.12127

Lau, B. H., Chan, C. L., \& Ng, S. M. (2020). Self-Compassion buffers the adverse mental health impacts of covid-19-related threats: Results from a cross-sectional survey at the first peak of Hong Kong's Outbreak. Frontiers in Psychiatry, 11, 585270. https:// doi.org/10.3389/fpsyt.2020.585270

Kaufman, E. A., Xia, M., Fosco, G., Yaptangco, M., Skidmore, C. R., \& Crowell, S. E. (2016). The Difficulties in Emotion Regulation Scale Short Form (DERS-SF): Validation and replication in adolescent and adult samples. Journal of Psychopathology and Behavioral Assessment, 38(3), 443-455. https://doi.org/10.1007/ s10862-015-9529-3

Karakasidou, E., Raftopoulou, G., Pezirkianidis, C., \& Stalikas, A. (2021). Validity, reliability and factorial structure of the Self Compassion Scale-Youth version in the Greek population. Psychology, 12, 536-553. https://doi.org/10.4236/psych.2021.124033

Khramtsova, I. I., \& Chuykova, T. S. (2016). Mindfulness and selfcompassion as predictors of humor styles in US and Russia. Social Psychology and Society, 7(2), 93-108. https://doi.org/10.17759/ sps.2016070207
Lathren, C., Bluth, K., \& Park, J. (2019). Adolescent self-compassion moderates the relationship between perceived stress and internalizing symptoms. Personality and Individual Differences, 143, 36-41. https://doi.org/10.1016/j.paid.2019.02.008

Lee, S. A. (2020). Coronavirus Anxiety Scale: A brief mental health screener for COVID-19 related anxiety. Death Studies, 44(7), 393-401. https://doi.org/10.1080/07481187.2020.1748481

Li, A., Wang, S., Cai, M., Sun, R., \& Liu, X. (2021). Self-compassion and life-satisfaction among Chinese self-quarantined residents during COVID-19 pandemic: A moderated mediation model of positive coping and gender. Personality and Individual Differences, 170(19), 110457. https://doi.org/10.1016/j.paid.2020. 110457

Lim, N. (2016). Cultural differences in emotion: Differences in emotional arousal level between the East and the West. Integrative Medicine Research, 5(2), 105-109. https://doi.org/10.1016/j.imr. 2016.03.004

Marsh, H. W., Liem, G. A. D., Martin, A. J., Morin, A. J. S., \& Nagengast, B. (2011). Methodological measurement fruitfulness of exploratory structural equation modeling (ESEM): New approaches to key substantive issues in motivation and engagement. Journal of Psychoeducational Assessment, 29(4), 322-346. https://doi.org/10.1177/0734282911406657

Marsh, I. C., Chan, S. W. Y., \& MacBeth, A. (2018). Self-compassion and psychological distress in adolescents - a meta-analysis. Mindfulness, 9(4), 1011-1027. https://doi.org/10.1007/ s12671-017-0850-7

Marsh, H. W., Morin, A. J., Parker, P. D., \& Kaur, G. (2014). Exploratory structural equation modeling: An integration of the best features of exploratory and confirmatory factor analysis. Annual Review of Clinical Psychology, 10, 85-110. https://doi.org/10. 1146/annurev-clinpsy-032813-153700

McKay, T., \& Walker, B. R. (2021). Mindfulness, self-compassion and wellbeing. Personality and Individual Differences, 168, 11041. https://doi.org/10.1016/j.paid.2020.1104122

Mona, S., \& Angela, J. (2018). Mindful self-compassion: How it can enhance resilience. Journal of Psychosocial Nursing and Mental Health Services, 56(1), 15-17. https://doi.org/10.3928/0279369520171219-01

Morale, S.,\& Fox N. A. (2019). A neuroscience perspective on emotional development. In: LoBue V., Pérez-Edgar K., \& Buss K. (eds) Handbook of emotional development (pp. 57-81), Cham: Springer. https://doi.org/10.1007/978-3-030-17332-6_4

Moreira, H., \& Canavarro, M. C. (2020). Mindful parenting is associated with adolescents' difficulties in emotion regulation through adolescents' psychological inflexibility and self-compassion. Journal of Youth and Adolescence, 49(1), 192-211. https://doi. org/10.1007/s10964-019-01133-9

Morin, A. J. S., Arens, K., Tran, A., \& Caci, H. (2016). Exploring sources of construct-relevant multidimensionality in psychiatric measurement: A tutorial and illustration using the composite scale of morningness. International Journal Methods Psychiatry Research., 25, 277-288. https://doi.org/10.1002/mpr.1485

Muris, P., Otgaar, H., López, A., Kurtic, I., \& van de Laar, I. (2020). The (non)protective role of self-compassion in internalizing symptoms: Two empirical studies in adolescents demonstrating unwanted effects of using the Self-Compassion Scale total score. Advance online publication. https://doi.org/10.1007/ s12671-020-01514-3

Muris, P., Otgaar, H., \& Petrocchi, N. (2016). Protection as the mirror image of psychopathology: Further critical notes on the SelfCompassion Scale. Mindfulness, 7(3), 787-790. https://doi.org/ 10.1007/s12671-016-0509-9

Muthén, L. K., \& Muthén, B. O. (1998-2012). Mplus user's guide statistical analysis with latent variables (7th ed.). Los Angeles, CA: Muthén \& Muthén. 
Nazari, N., \& Griffiths, M. D. (2020). Psychometric validation of the Persian version of the Emotional Style Questionnaire. Current Psychology. Advance online publication. https://doi.org/10.1007/ s12144-020-01205-1

Nazari, N., Zekiy, A. O., Feng, L. S., \& Griffiths, M. D. (2021). Psychometric validation of the Persian version of the COVID19-Related Psychological Distress Scale and association with COVID-19 Fear, COVID-19 anxiety, optimism, and lack of resilience. Advance online publication. https://doi.org/10.1007/ s11469-021-00540-z

Neff, K. D. (2003). Self-Compassion Scale. Self and Identity, 2, 223 250. https://doi.org/10.1080/15298860390209035

Neff, K. D. (2016). The Self-Compassion Scale is a valid and theoretically coherent measure of self-compassion. Mindfulness, 7(1), 264-274. https://doi.org/10.1007/s12671-015-0479-3

Neff, K. D., Bluth, K., Tóth-Király, I., Davidson, O., Knox, M. C., Williamson, Z., \& Costigan, A. (2021). Development and validation of the Self-Compassion Scale for Youth. Journal of Personality Assessment, 103(1), 92-105. https://doi.org/10.1080/00223891. 2020.1729774

Neff, K. D., \& McGehee, P. (2010). Self-compassion and psychological resilience among adolescents and young adults. Self and Identity, 9(3), 225-240. https://doi.org/10.1080/15298860902979307

Neff, K. D., Pisitsungkagarn, K., \& Hsieh, Y. P. (2008). Self-compassion and self-construal in the United States, Thailand, and Taiwan. Journal of Cross-Cultural Psychology, 39(3), 267-285. https:// doi.org/10.1177/0022022108314544

Neff, K. D., Rude, S. S., \& Kirkpatrick, K. L. (2007). An examination of self-compassion in relation to positive psychological functioning and personality traits. Journal of Research in Personality, 41(4), 908-916. https://doi.org/10.1016/j.jrp.2006.08.002

Neff, K. D., Tóth-Király, I., Yarnell, L. M., Arimitsu, K., Castilho, P., Ghorbani, N., ... Mantzios, M. (2019). Examining the factor structure of the Self-Compassion Scale in 20 diverse samples: Support for use of a total score and six subscale scores. Psychological Assessment, 31(1), 27-45. https://doi.org/10.1037/pas00 00629

Pechtel, P., \& Pizzagalli, D. A. (2011). Effects of early life stress on cognitive and affective function: An integrated review of human literature. Psychopharmacology (berl), 214(1), 55-70. https://doi. org/10.1007/s00213-010-2009-2

Pérez-Aranda, A., García-Campayo, J., Gude, F., Luciano, J. V., Feliu-Soler, A., González-Quintela, A., López-del-Hoyo, Y., \& Montero-Marin, J. (2021). Impact of mindfulness and self-compassion on anxiety and depression: The mediating role of resilience. International Journal of Clinical and Health Psychology, 21(2), 100229. https://doi.org/10.1016/j.ijchp.2021.100229

Pfabigan, D. M., Wucherer, A. M., Wang, X., Pan, X., Lamm, C., \& Han, S. (2018). Cultural influences on the processing of social comparison feedback signals-an ERP study. Social Cognitive and Affective Neuroscience, 13(12), 1317-1326. https://doi.org/ 10.1093/scan/nsy097

Phillips, W. J. (2019). Self-compassion mindsets: The components of the self-compassion scale operate as a balanced system within individuals. Current Psychology, 40(2020), 5040-5053. https:// doi.org/10.1007/s12144-019-00452-1

Potter, R. F., Yar, K., Francis, A. J. P., \& Schuster, S. (2014). Selfcompassion mediates the relationship between parentalcriticism and social anxiety. International Journal of Psychology and Psychological Therapy, 14(1), 33-43.

Pullmer, R., Chung, J., Samson, L., Balanji, S., \& Zaitsoff, S. (2019). A systematic review of the relation between self-compassion and depressive symptoms in adolescents. Journal of Adolescence, 74, 210-220. https://doi.org/10.1016/j.adolescence.2019.06.006

Pyszkowska, A. (2020). Personality predictors of self-compassion, egoresiliency and psychological flexibility in the context of quality of life. Personality and Individual Differences, 161, 109932. https:// doi.org/10.1016/j.paid.2020.109932

Raes, F. (2011). The effect of self-compassion on the development of depression symptoms in a non-clinical sample. Mindfulness, 2(1), 33-36. https://doi.org/10.1007/s12671-011-0040-y

Rammstedt, B., \& John, O. P. (2007). Measuring personality in one minute or less: A 10-item short version of the Big Five Inventory in English and German. Journal of Research in Personality, 41(1), 203-212. https://doi.org/10.1016/j.jrp.2006.02.001

Ramalho, S. M., Trovisqueira, A., de Lourdes, M., Gonçalves, S., Ribeiro, I., Vaz, A. R., et al. (2021). The impact of COVID-19 lockdown on disordered eating behaviors: the mediation role of psychological distress. Eating and Weight Disorders - Studies on Anorexia, Bulimia and Obesity. https://doi.org/10.1007/ s40519-021-01128-1

Roeser, R. W., \& Pinela, C. (2014). Mindfulness and compassion training in adolescence: A developmental contemplative science perspective. New Directions for Youth Development, 2014(142), 9-30. https://doi.org/10.1002/yd.20094

Schmitt, D. P., Long, A. E., McPhearson, A., O’Brien, K., Remmert, B., \& Shah, S. H. (2016). Personality and gender differences in global perspective. International Journal of Psychology, 52(s1), 45-56. https://doi.org/10.1002/ijop.12265

Shin, H. S., Black, D. S., Shonkoff, E. T., Riggs, N. R., \& Pentz, M. A. (2016). Associations among dispositional mindfulness, selfcompassion, and executive function proficiency in early adolescents. Mindfulness, 7(6), 1377-1384. https://doi.org/10.1007/ s12671-016-0579-8

Sekowski, M., Gambin, M., Cudo, A., Wozniak-Prus, M., Penner, F., Fonagy, P., \& Sharp, C. (2020). The relations between childhood maltreatment, shame, guilt, depression and suicidal ideation in inpatient adolescents. Journal of Affective Disorders, 276(2), 667-677. https://doi.org/10.1016/j.jad.2020.07.056

Shapira, L. B., \& Mongrain, M. (2010). The benefits of self-compassion and optimism exercises for individuals vulnerable to depression. Journal of Positive Psychology, 5(5), 377-389. https://doi. org/10.1080/17439760.2010.516763

Shattell, M., \& Johnson, A. (2018). Mindful self-compassion: How it can enhance resilience. Journal of Psychosocial Nursing and Mental Health Services, 56(1), 15-17. https://doi.org/10.3928/ 02793695-20171219-01

Smith, B. W., Dalen, J., Wiggins, K., Tooley, E., Christopher, P., \& Bernard, J. (2008). The Brief Resilience Scale: Assessing the ability to bounce back. International Journal of Behavioral Medicine, 15(3), 194-200. https://doi.org/10.1080/10705500802222972

Sutton, E., Schonert-Reichl, K. A., Wu, A. D., \& Lawlor, M. S. (2018). Evaluating the reliability and validity of the self-compassion scale short form adapted for children ages 8-12. Child Indicators Research, 11(4), 1217-1236. https://doi.org/10.1007/ s12187-017-9470-y

Tóth-Király, I., Bőthe, B., \& Orosz, G. (2017). Exploratory structural equation modeling analysis of the Self-Compassion Scale. Mindfulness, 8(4), 881-892. https://doi.org/10.1007/ s12671-016-0662-1

Tóth-Király, I., \& Neff, K. D. (2020). Is self-compassion universal? Assessment. Advance online publication. https://doi.org/10.1177/ 1073191120926232

Trompetter, H. R., de Kleine, E., \& Bohlmeijer, E. T. (2017). Why does positive mental health buffer against psychopathology? An exploratory study on self-compassion as a resilience mechanism and adaptive emotion regulation strategy. Cognitive Therapy and Research, 41(3), 459-468. https://doi.org/10.1007/ s10608-016-9774-0

Werner, K. H., Jazaieri, H., Goldin, P. R., Ziv, M., Heimberg, R. G., \& Gross, J. J. (2012). Self-compassion and social anxiety disorder. 
Anxiety, Stress and Coping, 25(5), 543-558. https://doi.org/10. 1080/10615806.2011.608842

Wilson, A. C., Mackintosh, K., Power, K., \& Chan, S. W. Y. (2018). Effectiveness of Self-Compassion related therapies: A systematic review and meta-analysis. Mindfulness, 10(6), 979-995. https:// doi.org/10.1007/s12671-018-1037-6

Wu, X., Nazari, N., \& Griffiths, M. D. (2021). Using fear and anxiety related to COVID-19 to predict cyberchondria: Cross-sectional survey study. Journal of Medical Internet Research, 23(6), e26285. https://doi.org/10.2196/26285

Yarnell, L. M., Neff, K. D., Davidson, O. A., \& Mullarkey, M. (2019). Gender differences in self-compassion: Examining the role of gender role orientation. Mindfulness, 10(6), 1136-1152.

Yarnell, L. M., Stafford, R. E., Neff, K. D., Reilly, E. D., Knox, M. C., $\&$ Mullarkey, M. (2015). Meta-analysis of gender differences in self-compassion. Self and Identity, 14(5), 499-520. https://doi.org /10.1080/15298868.2015.1029966

Vettese, L. C., Dyer, C. E., Li, W. L., \& Wekerle, C. (2011). Does self-compassion mitigate the association between childhood maltreatment and later emotion regulation difficulties? A preliminary investigation. International Journal of Mental Health and Addiction, 9(5), 480-491. https://doi.org/10.1007/s11469-011-9340-7

von Suchodoletz, A., \& Hepach, R. (2021). Cultural values shape the expression of self-evaluative social emotions. Scientific Reports, 11(1), 13169. https://doi.org/10.1038/s41598-021-92652-8

Zessin, U., Dickhäuser, O., \& Garbade, S. (2015). The relationship between self-compassion and well-being: A meta-analysis. Applied Psychology: Health and Well-Being, 7(3), 340-364. https://doi.org/10.1111/aphw.12051

Zeller, M., Yuval, K., Nitzan-Assayag, Y., \& Bernstein, A. (2015). Self-compassion in recovery following potentially traumatic stress: Longitudinal study of at-risk youth. Journal of Abnormal Child Psychology, 43(4), 645-653. https://doi.org/10.1007/ s10802-014-9937-y

Publisher's Note Springer Nature remains neutral with regard to jurisdictional claims in published maps and institutional affiliations. 